\title{
Dynamic Topological Logic of Metric Spaces
}

\author{
David Fernández-Duque \\ Group for Logic, Language and Information, \\ Universidad de Sevilla
}

October 11, 2010

\begin{abstract}
Dynamic Topological Logic $(\mathcal{D} \mathcal{T} \mathcal{L})$ is a modal framework for reasoning about dynamical systems, that is, pairs $\langle X, f\rangle$ where $X$ is a topological space and $f: X \rightarrow X$ a continuous function.

In this paper we consider the case where $X$ is a metric space. We first show that any formula which can be satisfied on an arbitrary dynamic topological system can be satisfied on one based on a metric space; in fact, this space can be taken to be countable and have no isolated points. Since any metric space with these properties is homeomorphic to the set of rational numbers, it follows that any formula can be satisfied on a system based on $\mathbb{Q}$.

We then show that the situation changes when considering complete metric spaces, by exhibiting a formula which is not valid in general but is valid on the class of systems based on a complete metric space. While we do not attempt to give a full characterization of the set of valid formulas on this class we do give a relative completeness result; any formula which is satisfiable on a dynamical system based on a complete metric space is also satisfied on one based on the Cantor space.
\end{abstract}

\section{Introduction}

Whether to study real-world phenomena or for purely theoretical purposes, dynamical systems appear once and again in many branches of mathematics. The precise meaning of 'dynamical system' may vary, but the basic intuition is that of a structure which changes over time. This structure is usually meant to represent space; in a broad sense we might take 'space' to be any topological space, in a narrow sense we may demand that it be Euclidean, and somewhere in the middle we can simply model it as a metric space.

We also have several candidates for modeling time. Perhaps the most natural candidates are $\mathbb{R}$ ('continuous time') and $\mathbb{N}$ ('discrete time'). Here we will focus on the latter interpretation, considering a dynamic topological system to be a topological space under the action of a continuous function $f$. A thorough treatment of the mathematics of such systems can be found in [1]. 
For the logical modeling of dynamic topological systems, we may borrow from temporal logic [8] and from spatial interpretations of modal logic [13]. The continuity of $f$ gives rise to a non-trivial interaction between the modalities, giving rise to the 'next-interior' logic S4C introduced in [2] and, later, to Dynamic Topological Logic $(\mathcal{D T} \mathcal{L})$, introduced in [7], adding a 'henceforth' modality to the language.

Dynamic Topological Logic has blossomed into a rich and fruitful research area. Variations in the semantics generate a complex jungle of logics; even $\mathcal{D} \mathcal{T} \mathcal{L}$ interpreted over the real line is not yet fully understood (but see $[7,10,11]$ ).

Here we will consider $\mathcal{D} \mathcal{T} \mathcal{L}$ over metric spaces. This gives us more restrictive semantics than the general topological interpretation; however, we will show the distinction is unimportant since any formula in the language of $\mathcal{D} \mathcal{T} \mathcal{L}$ which can be satisfied on a topological space can be satisfied on a metric space. Indeed, the latter can be taken to be a perfect ${ }^{1}$ countable metric space, giving us a sort of downward Löwenheim-Skolem theorem for $\mathcal{D} \mathcal{T} \mathcal{L}$. Further, all such spaces are homeomorphic, so we have completeness for any fixed perfect countable metric space, such as the set of rational numbers $\mathbb{Q}$, thus generalizing a result of Kremer [6].

On the other hand, $\mathbb{Q}$ cannot be replaced, say, by the Cantor set in the above statement, since we will also show that $\mathcal{D} \mathcal{T} \mathcal{L}$ is incomplete for interpretations on the class of complete metric spaces.

In general, if $X$ is a class of dynamic topological systems, we will write $\mathcal{D} \mathcal{T} \mathcal{L}_{\mathrm{X}}$ for the set of valid formulas of the language of $\mathcal{D} \mathcal{T} \mathcal{L}$ when interpreted on elements of $\mathrm{X}$, and similarly $\mathcal{D} \mathcal{T} \mathcal{L}_{\mathfrak{X}}$ will denote the set of valid formulas interpreted over all systems based on the space $\mathfrak{X}$. If $X$ is the class of all dynamical systems, we will write $\mathcal{D} \mathcal{T} \mathcal{L}$ instead of $\mathcal{D} \mathcal{T} \mathcal{L}_{\mathrm{S}}$.

An important part of the Dynamic Topological Logic project involves understanding $\mathcal{D} \mathcal{T} \mathcal{L}_{\mathrm{X}}$ for those classes $\mathrm{X}$ which are most relevant in the study of dynamical systems. Some classes of interest are

- the class DTS of all dynamical systems over a topological space;

- the class A of all dynamical systems based on an Aleksandroff space;

- the class $\mathrm{M}$ of all systems based on a metric space;

- the class CompM of all systems based on a complete metric space;

- all systems based on the rational numbers, $\mathbb{Q}$;

- all systems based on the real line, $\mathbb{R}$;

- all systems based on the complex plane $\mathbb{C}$ (or, equivalently, $\mathbb{R}^{2}$ ),

- all systems based on the Cantor set, $\mathbb{K}$.

In this paper we focus on some of those classes of systems involving metric spaces, namely $\mathrm{M}$, CompM, $\mathbb{Q}$ and $\mathbb{K}$. Our main results are the following:

\footnotetext{
${ }^{1}$ I.e., containing no isolated points.
} 
1. $\mathcal{D} \mathcal{T} \mathcal{L}=\mathcal{D} \mathcal{T} \mathcal{L}_{\mathbb{Q}}$,

2. $\mathcal{D} \mathcal{T} \mathcal{L} \neq \mathcal{D} \mathcal{T} \mathcal{L}_{\text {CompM }}$ and

3. $\mathcal{D} \mathcal{T} \mathcal{L}_{\text {CompM }}=\mathcal{D} \mathcal{T} \mathcal{L}_{\mathbb{K}}$.

Thus $\mathbb{Q}$ and $\mathbb{K}$ serve as 'universal spaces' for $M$ and CompM, respectively. These results advance a considerable body of knowledge by many authors:

1. On the next-interior fragment, the logic interpreted over DTS is equal to that over $\mathrm{A}[2], \mathbb{Q}[6], \mathbb{K}[9]$ (and hence over $\mathrm{M}$ and CompM) and $\mathbb{C}[4]$.

It is not, however, equal to the logic over $\mathbb{R}[7,10,11]$.

2. Over the full language, the logics over DTS, A, $\mathbb{R}$ and $\mathbb{C}$ are all distinct $[7,4]$.

We further have that $\mathcal{D} \mathcal{T} \mathcal{L}_{\mathbb{C}} \subsetneq \mathcal{D} \mathcal{T} \mathcal{L}_{\mathrm{A}}$ [4]. These results can be summarized in the following diagram:

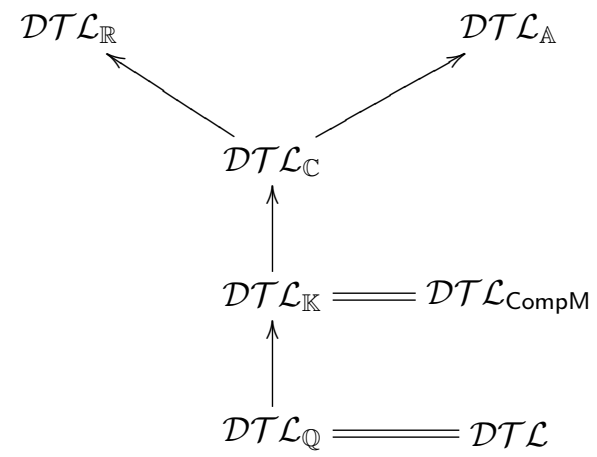

The arrows indicate proper inclusion.

The layout of this paper is as follows. In $\S 2$, we give an introduction to the syntax and semantics of $\mathcal{D} \mathcal{T} \mathcal{L}$. Then, $\S 3-4$ provide an overview of quasimodels, introduced in [5], where proofs of the results we mention may be found, and $\$ 7$ proves our first completeness result, namely, that $\mathcal{D} \mathcal{T} \mathcal{L}=\mathcal{D} \mathcal{T} \mathcal{L}_{\mathfrak{D}}$ for any perfect countable metric space $\mathfrak{D}$. Then, $\S 8$ shows that $\mathcal{D} \mathcal{T} \mathcal{L} \neq \mathcal{D} \mathcal{T} \mathcal{L}_{\text {CompM }}$ by exhibiting a formula which is not valid in general but is valid on the class of dynamic topological systems based on a complete metric space. Finally, $\S 9$ shows that any formula satisfiable on a system based on a complete metric space can be satisfied on one based on the Cantor space.

\section{Dynamic Topological Logic}

We will work in a language whose formulas are built from propositional variables in a countably infinite set PV using the Boolean connectives $\wedge$ and $\neg$ (all other 
connectives are to be defined in terms of these) and the unary modal operators $\square$ ('interior'), $(f)$ ('next') and $[f]$ ('henceforth'). We write $\diamond$ as a shorthand for $\neg \square \neg$ and $\langle f\rangle$ for $\neg[f] \neg$. Semantics are given by dynamical systems over topological spaces, or dynamic topological systems.

Definition 2.1 (dynamic topological systems/models). A dynamic topological system is a triple

$$
\mathfrak{X}=\left\langle|\mathfrak{X}|, \mathcal{T}_{\mathfrak{X}}, f_{\mathfrak{X}}\right\rangle,
$$

where $\left\langle|\mathfrak{X}|, \mathcal{T}_{\mathfrak{X}}\right\rangle$ is a topological space and

$$
f_{\mathfrak{X}}:|\mathfrak{X}| \rightarrow|\mathfrak{X}|
$$

is continuous.

$A$ valuation on $\mathfrak{X}$ is an assignment of a set $p_{\mathfrak{X}} \subseteq|\mathfrak{X}|$ to each $p \in \mathrm{PV}$. A dynamic topological model (DTM) is a dynamic topological system equipped with a valuation $\vec{p}_{\mathfrak{X}}$.

We can use $\vec{p}_{\mathfrak{X}}$ to interpret all formulas of $\mathcal{D} \mathcal{T} \mathcal{L}$ as subsets of $|\mathfrak{X}|$ :

Definition 2.2 (Valuation; validity). Given a dynamic topological model $\mathfrak{X}$, we assign a set $\llbracket \varphi \rrbracket_{\mathfrak{X}} \subseteq|\mathfrak{X}|$ to each formula $\varphi \in \mathcal{L}$ as follows:

$$
\begin{array}{ll}
\llbracket p \rrbracket_{\mathfrak{X}} & =p_{\mathfrak{X}} ; \\
\llbracket \alpha \wedge \beta \rrbracket_{\mathfrak{X}} & =\llbracket \alpha \rrbracket_{\mathfrak{X}} \cap \llbracket \beta \rrbracket_{\mathfrak{X}} \\
\llbracket \neg \alpha \rrbracket_{\mathfrak{X}} & =|\mathfrak{X}| \backslash \llbracket \alpha \rrbracket_{\mathfrak{X}} \\
\llbracket \square \alpha \rrbracket_{\mathfrak{X}} & =\llbracket \alpha \rrbracket_{\mathfrak{X}}^{\circ} \\
\llbracket(f) \alpha \rrbracket_{\mathfrak{X}} & =f_{\mathfrak{X}}^{-1} \llbracket \alpha \rrbracket_{\mathfrak{X}} \\
\llbracket[f] \alpha \rrbracket_{\mathfrak{X}} & =\bigcap_{n \geq 0} f_{\mathfrak{X}}^{-n} \llbracket \alpha \rrbracket_{\mathfrak{X}} .
\end{array}
$$

We say a formula $\varphi$ is valid on $\mathfrak{X}$ if $\llbracket \varphi \rrbracket=|\mathfrak{X}|$, and write $\mathfrak{X} \models \varphi$.

Similarly, if $\mathbf{X}$ is a class of dynamic topological systems, we write $\mathrm{X} \models \varphi$ if $\mathfrak{X}=\varphi$ for every $\mathfrak{X} \in \mathrm{X}$.

\section{Quasimodels}

This section along with $\S 4$ give a review of some notions from [5], although following the notation and terminology of [3]. We refer the reader to [5] for missing proofs.

We will define quasimodels for $\mathcal{D} \mathcal{T} \mathcal{L}$. These will be birelational Kripke models where each world is assigned a type, that is, a set of formulas which it 'intends' to satisfy. 
Denote the set of subformulas of $\varphi$ by $\operatorname{sub}(\varphi)$, and define

$$
\operatorname{sub}_{ \pm}(\varphi)=\operatorname{sub}(\varphi) \cup \neg \operatorname{sub}(\varphi) .
$$

We will treat $\operatorname{sub}_{ \pm}(\varphi)$ as if it were closed under negation, by implicitly identifying $\psi$ with $\neg \neg \psi$.

Definition 3.1 (type). A set of formulas $\Phi \subseteq \operatorname{sub}_{ \pm}(\varphi)$ is a $\varphi$-type if, for all $\psi \in \operatorname{sub}_{ \pm}(\varphi)$

$$
\psi \notin \Phi \Leftrightarrow \neg \psi \in \Phi
$$

and for all $\psi_{1} \wedge \psi_{2} \in \operatorname{sub}_{ \pm}(\varphi)$,

$$
\psi_{1} \wedge \psi_{2} \in \Phi \Leftrightarrow \psi_{1} \in \Phi \text { and } \psi_{2} \in \Phi \text {. }
$$

The set of $\varphi$-types will be denoted by type $(\varphi)$.

Definition 3.2 (typed preorder). Let $\varphi$ be a formula in the language of $\mathcal{D} \mathcal{T}$ and $S$ a set preordered by $\preccurlyeq$.

$A$ typing function on $S$ is an assignment

$$
t: S \rightarrow \operatorname{type}(\varphi)
$$

such that, for all $\square \psi \in \operatorname{sub}_{ \pm}(\varphi)$ and $w \in S, \square \psi \in t(w)$ if and only if $\psi \in t(v)$ whenever $v \preccurlyeq w$.

This is equivalent to the dual condition that $\nabla \psi \in t(w)$ if and only if there exists $v \preccurlyeq w$ such that $\psi \in t(v)$.

The main difference between quasimodels and standard models is that the dynamics on quasimodels are represented by a continuous relation rather than a function; here we will use a simple definition of a continuous relation as one for which preimages of open sets are open (note that this is not the standard definition). In order for this interpretation to be sound, however, we must place syntactic restrictions on these relations.

Definition 3.3 (sensible relation). Let $\varphi$ be a formula of $\mathcal{D} \mathcal{T} \mathcal{L}$ and $\Phi, \Psi \in$ type $(\varphi)$. The ordered pair $\langle\Phi, \Psi\rangle$ is sensible if

1. for all $(f) \psi \in \operatorname{sub}(\varphi),(f) \psi \in \Phi \Leftrightarrow \psi \in \Psi$ and

2. for all $[f] \psi \in \operatorname{sub}(\varphi),[f] \psi \in \Phi \Leftrightarrow(\psi \in \Phi$ and $[f] \psi \in \Psi)$.

Likewise, a pair $(w, v)$ of worlds in a typed preorder $\mathfrak{A}$ is sensible if $\langle t(w), t(v)\rangle$ is sensible.

A continuous relation

$$
\mapsto \subseteq|\mathfrak{A}| \times|\mathfrak{A}|
$$

is sensible if, for every $w \in|\mathfrak{A}|$,

$$
\{v: w \mapsto v\} \neq \varnothing
$$

and every pair in $\mapsto$ is sensible.

Further, $\mapsto$ is $\omega$-sensible if for all $\langle f\rangle \psi \in \operatorname{sub}_{ \pm}(\varphi)$,

$$
\langle f\rangle \psi \in t(w) \Leftrightarrow \exists v \in|\mathfrak{A}| \text { and } N \geq 0 \text { such that } \psi \in t(v) \text { and } w \mapsto^{N} v \text {. }
$$


Definition 3.4 (Quasimodel). A $\varphi$-quasimodel is a tuple

$$
\mathfrak{A}=\left\langle|\mathfrak{A}|, \preccurlyeq \mathfrak{A}, \mapsto_{\mathfrak{A}}, t_{\mathfrak{A}}\right\rangle,
$$

where $\left\langle|\mathfrak{A}|, \preccurlyeq \mathfrak{A}, t_{\mathfrak{A}}\right\rangle$ is a $\varphi$-typed Kripke frame and $\mapsto_{\mathfrak{A}}$ is an $\omega$-sensible relation on $|\mathfrak{A}|$.

$\mathfrak{A}$ satisfies $\varphi$ if there exists $w_{*} \in|\mathfrak{A}|$ such that $\varphi \in t_{\mathfrak{A}}\left(w_{*}\right)$.

We omit the subindex $\mathfrak{A}$ and write $\preccurlyeq, \mapsto$ instead of $\preccurlyeq \mathfrak{A}, \mapsto \mathfrak{A}$, except when this may lead to confusion.

\section{Generating dynamic topological models from quasimodels}

Given a $\varphi$-quasimodel $\mathfrak{A}$, we can construct a dynamic topological model $\lim \mathfrak{A}$ satisfying the same subformulas of $\varphi$; the points of $|\lim \mathfrak{A}|$ will not be worlds in $|\mathfrak{A}|$, but rather infinite $\mapsto$-paths.

\subsection{Realizing sequences}

A path in $\mathfrak{A}$ is any sequence $\left\langle w_{n}\right\rangle_{n<N}$, with $N \leq \omega$, such that $w_{n} \mapsto w_{n+1}$.

The continuity of $\mapsto$ has a natural generalization for finite paths. The following Lemma is proven in [5]:

Lemma 4.1. Let $\mathfrak{A}$ be a $\varphi$-quasimodel, $\left\langle w_{n}\right\rangle_{n \leq N}$ a finite path and $v_{0}$ be such that $v_{0} \preccurlyeq w_{0}$.

Then, there exists a path $\left\langle v_{n}\right\rangle_{n \leq N}$ such that, for $n \leq N, v_{n} \preccurlyeq w_{n}$.

Proof. This follows from an easy induction on $N$ using the cotinuity of $\mapsto$.

An infinite path $\vec{w}=\left\langle w_{n}\right\rangle_{n<\omega}$ is realizing if for all $n<\omega$ and $\langle f\rangle \psi \in t\left(w_{n}\right)$ there exists $K \geq n$ such that $\psi \in t\left(w_{K}\right)$.

Denote the set of realizing paths by $\overrightarrow{\mid \mathfrak{A}} \mid$. Note that

$$
\left.\overrightarrow{\mid \mathfrak{A}}|\subseteq| \mathfrak{A}\right|^{\mathbb{N}}
$$

if we view $|\mathfrak{A}|$ as a topological space with the preorder topology, then $|\mathfrak{A}|^{\mathbb{N}}$ naturally acquires the product topology. Consequently, $\overrightarrow{\mathfrak{A} \mid}$ can be seen as a topological space under the corresponding subspace topology; this topology on $\overrightarrow{\mid \mathfrak{A}} \mid$ will be denoted $\mathcal{T}_{\mathfrak{A}}$.

For $\vec{w}, \vec{v} \in \mid \overrightarrow{\mathfrak{A} \mid}$ and $N<\omega$, write $\vec{v} \preccurlyeq \stackrel{N}{\preccurlyeq}$ if $v_{n} \preccurlyeq w_{n}$ for all $n<N$. Then define

$$
\downarrow_{N}(\vec{w})=\{\vec{v} \in|\overrightarrow{\mathfrak{A}}|: \vec{v} \stackrel{N}{\preccurlyeq} \vec{w}\} .
$$

Sets of the form $\downarrow_{N}(\vec{w})$ form a basis for $\mathcal{T}_{\mathfrak{A}}[5]$. 


\subsection{Limit models}

We can define dynamics on $\overrightarrow{|\mathfrak{A}|}$ by the shift operator $\sigma$, given by

$$
\sigma\left(\left\langle w_{n}\right\rangle_{n<\omega}\right)=\left\langle w_{n+1}\right\rangle_{n<\omega} .
$$

This simply removes the first element in the sequence. The function $\sigma$ is continuous with respect to $\mathcal{T}_{\mathfrak{A}}$.

We can also use $t$ to define a valuation: if $p$ is a propositional variable, set

$$
p_{\mathfrak{A}}=\left\{\vec{w} \in \overrightarrow{|\mathfrak{A}|}: p \in t\left(w_{0}\right)\right\} .
$$

We are now ready to assign a dynamic topological model to every $\varphi$-quasimodel:

Definition 4.1 (limit model). Given a non-deterministic quasimodel $\mathfrak{A}$, define

$$
\lim \mathfrak{A}=\left\langle\overrightarrow{\mid \mathfrak{A}} \mid, \mathcal{T}_{\mathfrak{A}}, \sigma, \vec{p}_{\mathfrak{A}}\right\rangle
$$

to be the limit model of $\mathfrak{A}$.

Limit models are useful because of the following result, proven in [5]:

Proposition 4.1. Any satisfiable formula $\varphi$ can be satisfied on the limit model of a locally finite $\varphi$-quasimodel.

\section{Presimulations}

A powerful tool for comparing dynamic topological models (and models of modal logics in general) is that of a bisimulation, because bisimulations preserve the valuation of formulas. For $\mathcal{D} \mathcal{T} \mathcal{L}$ we will use bisimulations of the following form:

Definition 5.1 (Dynamic topological bisimulation). $A$ bisimulation between DTM's $\mathfrak{X}, \mathfrak{Y}$ is a continuous, open function

$$
\beta:|\mathfrak{X}| \rightarrow|\mathfrak{Y}|
$$

such that $f_{\mathfrak{Y}} \beta=\beta f_{\mathfrak{X}}$ and, for every propositional variable $p$,

$$
\llbracket p \rrbracket_{\mathfrak{X}}=\beta^{-1} \llbracket p \rrbracket_{\mathfrak{X}} .
$$

We then have the following:

Proposition 5.1. If $\beta$ is a bisimulation between DTM's $\mathfrak{X}$ and $\mathfrak{Y}$ and $\varphi$ is an arbitrary formula, then

$$
\llbracket \varphi \rrbracket_{\mathfrak{X}}=\beta^{-1} \llbracket \varphi \rrbracket_{\mathfrak{X}} .
$$

Proof. The proof follows by a fairly standard induction on the build of $\varphi$.

Bisimulations give us a general criterion for guaranteeing that a DTM $\mathfrak{X}$ is 'richer' than a DTM $\mathfrak{Y}$ in the sense that at least as many formulas of $\mathcal{D} \mathcal{T} \mathcal{L}$ can be satisfied on $\mathfrak{X}$ as on $\mathfrak{Y}$. 
Definition 5.2 (Richness). A presimulation between dynamic topological systems $\mathfrak{X}$ and $\mathfrak{Y}$ is a continuous, open, surjective function $\beta:|\mathfrak{X}| \rightarrow|\mathfrak{Y}|$ such that

$$
f_{\mathfrak{Y}} \beta=\beta f_{\mathfrak{X}} .
$$

If there exists a presimulation between $\mathfrak{X}$ and $\mathfrak{Y}$, we will say $\mathfrak{X}$ is at least as rich as $\mathfrak{Y}$ and write $\mathfrak{X} \gg \mathfrak{Y}$.

Lemma 5.1. If $\mathfrak{X} \gg \mathfrak{Y}$, then $\mathcal{D} \mathcal{T} \mathcal{L}_{\mathfrak{X}} \subseteq \mathcal{D} \mathcal{T} \mathcal{L}_{\mathfrak{Y}}$.

Proof. We will show that any formula $\varphi$ satisfiable on $\mathfrak{Y}$ is also satisfiable on $\mathfrak{X}$.

Let $\beta:|\mathfrak{X}| \rightarrow|\mathfrak{Y}|$ be a presimulation, and let $\llbracket \cdot \rrbracket_{\mathfrak{Y}}$ be a valuation on $\mathfrak{Y}$ such that $\llbracket \varphi \rrbracket_{\mathfrak{Y}} \neq \varnothing$.

Then, setting $\llbracket \cdot \rrbracket_{\mathfrak{X}}=\beta^{-1} \llbracket \cdot \rrbracket_{\mathfrak{Y}}$, we have by Proposition 5.1 that $\llbracket \varphi \rrbracket_{\mathfrak{X}}=$ $\beta^{-1} \llbracket \varphi \rrbracket_{\mathfrak{Y}}$. Since $\beta$ is surjective and $\llbracket \varphi \rrbracket_{\mathfrak{Y}} \neq \varnothing$, it follows that $\llbracket \varphi \rrbracket_{\mathfrak{X}} \neq \varnothing$, as desired.

\section{Metrizable topological spaces}

In this section we will review some of the fundamental properties of the class of metrizable spaces. In general it is convenient to work with metrizable rather than metric spaces since committing to a specific metric is sometimes counterproductive.

First a fact about countable spaces that some may find a bit surprising (the author certainly did):

Proposition 6.1 (Sierpinski, 1920). Any two perfect countable metric spaces are isomorphic to each other.

Proof. We will not present a proof here, but it basically follows a back-and-forth argument, passing from partial isomorphisms to a full isomorphism.

Lemma 6.1. If $\langle X, \mathcal{T}\rangle$ is metrizable, then it admits a bounded metric, i.e., the topology $\mathcal{T}$ can be induced by a metric $d$ satisfying $d(x, y)<1$ for all $x, y \in \mathcal{T}$.

Proof. Let $d^{\prime}$ be a metric on $X$ inducing $\mathcal{T}$. Then, consider

$$
d(x, y)=\frac{d^{\prime}(x, y)}{1+d^{\prime}(x, y)} .
$$

It is not hard to check that $d$ is a new metric on $X$, that it is bounded by 1 , and that $d$ also induces $\mathcal{T}$, as desired.

Proposition 6.2. Suppose $\left\langle\mathfrak{X}_{i}\right\rangle_{i<\omega}$ is a sequence of metrizable spaces. Then,

$$
\text { 1. } \prod_{i<\omega} \mathfrak{X}_{i} \text { and }
$$




\section{2. $\prod_{i \ll \infty} x_{i}$}

are metrizable.

Proof. We will show this by defining appropriate metrics on the two spaces. By Lemma 6.1, we can assume that each $\mathfrak{X}_{i}=\left\langle X_{i}, d_{i}\right\rangle$, where $d_{i}<1$.

Then, define

1. if $\vec{x}, \vec{y} \in \prod_{i<\omega} X_{i}, \vec{x}=\left\langle x_{i}\right\rangle_{i<\omega}$ and $\vec{y}=\left\langle y_{i}\right\rangle_{i<\omega}$, set

$$
d(\vec{x}, \vec{y})=\sum_{i<\omega} \frac{d_{i}\left(x_{i}, y_{i}\right)}{2^{i}}
$$

2. if $x, y \in \coprod_{i<\omega} X_{i}$, set

$$
d(x, y)= \begin{cases}d_{i}(x, y) & \text { if } x, y \in X_{i} \\ 1 & \text { otherwise }\end{cases}
$$

The reader can then check that these are metrics that induce the desired topologies. Note that the disjoint union does not need to be countable, but we will only need it in this form.

If $\langle X, d\rangle$ is a metric space, we will use the notation

$$
B_{\varepsilon}(x)=\{y \in X: d(x, y)<\varepsilon\} .
$$

Sets of this form form a basis for the topology on $\langle X, d\rangle$, even if we only consider rational $\varepsilon$. From the latter it follows that any countable metric space is also second-countable, i.e., has a countable basis.

\section{Rational completeness}

In this section we will show that $\mathcal{D} \mathcal{T} \mathcal{L}=\mathcal{D} \mathcal{T} \mathcal{L}_{\mathbb{Q}}$. Let us begin by showing how one can find countable models in a more general setting.

Proposition 7.1. Given any DTM $\mathfrak{X}$ based on a second-countable space there exists a countable set $Y \subseteq|\mathfrak{X}|$ such that $\mathfrak{X}\lceil Y$ satisfies the same set of formulas as $\mathfrak{X}$.

Proof. Let $\mathfrak{X}$ be a DTM based on a second-countable space.

Let $\mathcal{B}$ be a countable basis for $\mathcal{T}_{\mathfrak{X}}$.

Say a pair $\langle\varphi, B\rangle$ is a occurrence on $\mathfrak{X}$ if $\varphi$ is a formula, $B \in \mathcal{B}$ and $\llbracket \varphi \rrbracket_{\mathfrak{X}} \cap B$.

Let $\Lambda$ be the set of all occurrences on $\mathfrak{X}$. Note that $\Lambda$ is countable, since both our language and $\mathcal{B}$ are countable.

Choose a function $x$. $\Lambda \rightarrow|\mathfrak{X}|$ assigning to each $\lambda=\langle\varphi, B\rangle \in \Lambda$ a unique element $x_{\lambda}$ such that $x_{\lambda} \in \llbracket \varphi \rrbracket_{\mathfrak{X}}$. 
Let $Y_{0}=\left\{x_{\lambda}: \lambda \in \Lambda\right\}$ and let $Y$ be the orbit of $Y_{0}$ under $f_{\mathfrak{X}}$.

Clearly $Y_{0}$, and hence $Y$, is countable.

It remains to show that $\mathfrak{Y}=\mathfrak{X} \uparrow Y$ satisfies the same set of formulas as $\mathfrak{X}$. For this we will first show that, given any formula $\varphi$,

$$
\llbracket \varphi \rrbracket_{\mathfrak{Y}}=\llbracket \varphi \rrbracket_{\mathfrak{X}} \cap Y .
$$

This follows by a straightforward induction on formulas of which only the case of $\varphi=\square \psi$ is interesting.

Suppose, first, that $x \in \llbracket \square \psi \rrbracket_{\mathfrak{X}}$. This means that there exists a neighborhood $U$ of $x$ such that $U \subseteq \llbracket \psi \rrbracket_{\mathfrak{X}}$. By induction hypothesis, we have that $\llbracket \psi \rrbracket_{\mathfrak{Y}}=$ $\llbracket \psi \rrbracket_{\mathfrak{X}} \cap Y$, so $U \cap Y$ is a neighborhood of $x$ in $Y$ such that every point satisfies $\psi$, that is, $x \in \llbracket \square \psi \rrbracket_{\mathfrak{Y}}$.

Now, suppose that $x \notin \llbracket \square \psi \rrbracket_{\mathfrak{X}}$. This means that every neighborhood $U$ of $x$ contains at least one point not satisfying $\psi$.

Let $U \cap Y$ be any neighborhood of $x$ in $\mathfrak{Y}$ and $B$ be a basic set such that $x \in B \subseteq U$. Since $B$ contains a point not satisfying $\psi$, and hence satisfying $\neg \psi$, we have that $\lambda=\langle\neg \psi, B\rangle$ is a occurrence on $\mathfrak{X}$ and hence we have $x_{\lambda} \in$ $Y \cap B \cap \llbracket \neg \psi \rrbracket_{\mathfrak{X}}$. By induction hypothesis $x_{\lambda} \in \llbracket \neg \psi \rrbracket_{\mathfrak{Y}}$, and since $U$ was arbitrary we conclude that $x \notin \llbracket \psi \rrbracket_{\mathfrak{Y}}$.

Then, if a formula $\varphi$ is satisfied on $x \in|\mathfrak{X}|$, setting $\lambda=\langle\varphi, B\rangle$, where $B$ is any neighborhood of $x$, we have that $x_{\lambda} \in \llbracket \varphi \rrbracket_{\mathfrak{Y}}$.

Proposition 7.2. Given any finite topological space $\mathfrak{W}, \mathbb{Q} \gg \mathfrak{W}$.

Proof. This follows from the proof of the McKinsey-Tarski theorem of completeness of S4 for any perfect second-countable metric space [13].

Corollary 7.1. Given any countable, locally finite topological space $\mathfrak{W}$ and $w \in|\mathfrak{W}|, \mathbb{Q} \gg \mathfrak{W}$.

Proof. If $|\mathfrak{W}|$ is finite, this is just Proposition 7.2.

Otherwise, $|\mathfrak{W}|$ is properly infinite and we can write it as $\left\langle w_{n}\right\rangle_{n<\omega}$. For each $n<\omega$, by Proposition 7.2, we have a sequence of presimulations $\beta_{n}: \mathbb{Q} \rightarrow \downarrow w_{n}$, and this in turn gives us a map $\beta: \coprod_{n<\omega} \mathbb{Q} \rightarrow|\mathfrak{W}|$ given by $\coprod_{n<\omega} \beta_{n}$. The latter map is clearly a presimulation, and by Proposition $6.1 \coprod_{n<\omega} \beta_{n} \simeq \mathbb{Q}$, as desired.

Proposition 7.3. Given a locally finite $\varphi$-quasimodel $\mathfrak{A}$, there exists a dynamic topological system $\mathfrak{S}$ based on a second-countable metric space such that $\mathfrak{S} \gg$ $\lim \mathfrak{A}$.

Proof. By Corollary 7.1, we have a presimulation $\beta: \mathbb{Q} \rightarrow|\mathfrak{A}|$.

This induces a map $\vec{\beta}: \mathbb{Q}^{\mathbb{N}} \rightarrow|\mathfrak{A}|$ given by

$$
\vec{\beta}\left\langle x_{n}\right\rangle_{n<\omega}=\left\langle\beta\left(x_{n}\right)\right\rangle_{n<\omega} .
$$

One can check that this map is continuous and open. 
Then consider $S=\vec{\beta}^{-1}(|\overrightarrow{\mathfrak{A}}|)$ and $\gamma=\vec{\beta}\lceil S$.

In general, restrictions of continuous, open maps are still continuous and open, so $\gamma$ is continuous and open.

Further, $\sigma_{S}$ is a continuous function on $S$ which clearly satisfies

$$
\gamma \sigma_{S}=\sigma_{\mathfrak{A}} \gamma
$$

Setting $\mathfrak{S}=\left\langle\mathbb{Q}^{\mathbb{N}} \mid S, \sigma_{S}\right\rangle$, we have that $\mathfrak{S} \gg \lim \mathfrak{A}\left(\right.$ via $\gamma$ ). Since $\mathbb{Q}^{\mathbb{N}}$ is metrizable and second-countable, so is $\mathbb{Q}^{\mathbb{N}} \uparrow S$, as desired.

Proposition 7.4. Given a DTS $\mathfrak{X}$ based on a countable metrizable space, there exists a DTS $\mathfrak{Y}$ based on a perfect countable metric space such that $\mathfrak{Y} \gg \mathfrak{X}$.

Proof. Let $\mathfrak{X}$ be a DTS based on a countable metric space.

Define $|\mathfrak{Y}|=|\mathfrak{X}| \times \mathbb{Q}$ with the product topology, $f_{\mathfrak{Y}}(x, r)=\left\langle f_{\mathfrak{X}}(x), r\right\rangle$ and let $\beta$ be the projection onto the first component.

Then clearly $\mathfrak{Y}$ is a DTS based on a perfect countable metric space, and $\mathfrak{Y} \gg \mathfrak{X}$ via $\beta$.

Theorem 7.1. Any formula $\varphi$ which is satisfiable can be satisfied on a DTM based on a perfect countable metric space.

Further, $\mathcal{D} \mathcal{T} \mathcal{L}_{\mathrm{M}}$ is strongly complete for such a space in the sense that any set of formulas that can be satisfied on a single point of a metric space an be satisfied on a point on a perfect countable metric space.

Proof. If a set of formulas $\Phi$ is satisfiable, it can be satisfied on $\lim \mathfrak{A}$ for some countable, locally finite $\varphi$-quasimodel $\mathfrak{A}$. Then, by Proposition 7.3, there exists a DTM $\mathfrak{X}$ based on a second-countable metric space such that $\mathfrak{X}$ satisfies $\Phi$. By Proposition 7.1 there is $\mathfrak{Y} \leq \mathfrak{X}$ based on a countable space (thus on a countable metric space) satisfying $\Phi$ as well, and by Proposition 7.4 there is $\mathfrak{Z} \gg \mathfrak{Y}$ based on a perfect countable metric space, so that $\Phi$ can be satisfied on $\mathfrak{Z}$ as well, as desired.

Corollary 7.2. $\mathcal{D} \mathcal{T} \mathcal{L}=\mathcal{D} \mathcal{T} \mathcal{L}_{\mathbb{Q}}$

Proof. Any two perfect countable metric spaces are isomorphic to each other by Proposition 6.1, so this is a straightforward consequence of Theorem 7.1.

\section{Incompleteness of $\mathcal{D} \mathcal{T} \mathcal{L}$ for complete metric spaces}

In [4] it was shown that $\mathcal{D} \mathcal{T} \mathcal{L}$ is not complete for $\mathbb{R}^{2}$ by exhibiting a formula which is valid on the class of dynamical systems based on a locally connected space but not valid in general. This shows that $\mathcal{D} \mathcal{T} \mathcal{L}$ is incomplete for interpretations based on $\left\{\mathbb{R}^{n}: n<\omega\right\}$, on Hilbert or Banach spaces, etc. However, it does not say anything about completeness for interpretations based on the Cantor space, which is totally disconnected, but shares with the above spaces 
the property of being complete as a metric space. Indeed, it was shown in [11] that $\mathcal{D} \mathcal{T} \mathcal{L}_{\mathrm{H}} \neq \mathcal{D} \mathcal{T} \mathcal{L}_{\text {CompMH }}$ by exhibiting a formula which was valid on the latter but not on the former, where $\mathrm{H}$ is the class of all dynamical systems $\mathfrak{X}$ where $f_{\mathfrak{X}}$ is a homeomorphism and CompMH is subclass of $\mathrm{H}$ whose systems are based on a complete metric space.

In this section we will show that the same holds over the class of all spaces, that is,

$$
\mathcal{D} \mathcal{T} \mathcal{L} \neq \mathcal{D} \mathcal{T} \mathcal{L}_{\text {CompM }}
$$

by a similar argument, showing a formula which is contained only on the righthand side and, in fact, for a very similar reason. However, because $\mathcal{D} \mathcal{T} \mathcal{L}$ is weaker than $\mathcal{D} \mathcal{T} \mathcal{L}_{\mathrm{H}}$, the formula we must use is a bit more complex.

Recall that the Baire Category Theorem reads as follows:

Theorem 8.1 (Baire Category Theorem). Let $\langle X, d\rangle$ be a complete metric space and $\left\{U_{n}\right\}_{n \geq 0}$ a collection of dense, open sets. Then,

$$
\bigcap_{n \geq 0} U_{n}
$$

is dense as well.

We will construct a formula $\varphi$ which expresses a form of this theorem.

Consider the following formulas:

$$
\begin{aligned}
& \varphi_{0}=\diamond s \wedge \diamond \neg s \\
& \varphi_{1}=s \leftrightarrow(f) s \\
& \varphi_{2}=p \rightarrow \square p \\
& \varphi_{3}=s \wedge \neg p \rightarrow \square(\neg s \rightarrow p)
\end{aligned}
$$

and define

$$
\text { Baire }=\square[f] \bigwedge_{n \leq 3} \varphi_{n} \rightarrow \diamond[f] p .
$$

Proposition 8.1. Baire is not valid over the class of all DTM's.

Proof. We will show this two different ways, one by exhibiting an actual DTM refuting Baire, and the other using quasimodels.

1. Consider a model $\mathfrak{X}$ over $\mathbb{Q}$ with $f_{\mathfrak{X}}(x)=x+1$.

Let $\left\langle r_{n}\right\rangle_{n<\omega}$ be an enumeration of $\mathbb{Q} \cap(-1 / 2,1 / 2)$ and set

$$
\llbracket p \rrbracket_{\mathfrak{X}}=\left\{r_{n}+k: k<n<\omega\right\} .
$$

Pick any set of rationals $S \subseteq(-1 / 2,1 / 2)$ which is dense but contains no open balls and then set $\llbracket s \rrbracket_{\mathfrak{X}}=S+\mathbb{Z}$ (for example, $S$ can be the set of all rationals between $-1 / 2$ and $1 / 2$ of the form $\left.m / 2^{n}\right)$.

We claim that $0 \notin \llbracket$ Baire $\rrbracket_{\mathfrak{X}}$. 
To see this, first note that $(-1 / 2,1 / 2) \cap \llbracket[f] p \rrbracket_{\mathfrak{X}}=\varnothing$. The reason for this is that, given $x \in \mathbb{Q} \cap(-1 / 2,1 / 2)$, we have $x=r_{n}$ for some value of $n$ and hence $f_{\mathfrak{X}}^{n}(x) \notin \llbracket p \rrbracket_{\mathfrak{X}}$.

This of course shows that $0 \notin \llbracket \triangleright[f] p \rrbracket_{\mathfrak{X}}$.

Meanwhile, let us check that

$$
\mathbb{Q} \cap(-1 / 2,1 / 2) \subseteq \llbracket \square[f] \varphi_{i} \rrbracket_{\mathfrak{X}}
$$

for all $i \leq 3$. Actually, it turns out that all of $\mathbb{Q}$ satisfies each $\varphi_{i}$, which is what we will prove.

$\varphi_{0}$ : This formula says that both $\llbracket s \rrbracket_{\mathfrak{X}}$ and $\llbracket \neg s \rrbracket_{\mathfrak{X}}$ are dense, which is true on all $\mathbb{Q}$ by the way we selected $\llbracket s \rrbracket_{\mathfrak{X}}$, i.e., as the orbit of a set which is dense in $(-1 / 2,1 / 2)$ but contains no open interval.

$\varphi_{1}$ : Once again this formula is true on all $\mathbb{Q}$ by the way we selected $\llbracket s \rrbracket_{\mathfrak{X}}$ simply because it is of the form $S+\mathbb{Z}$ (i.e., a point satisfies $s$ if and only if every other point on its orbit does).

$\varphi_{2}$ : This holds whenever $\llbracket p \rrbracket_{\mathfrak{X}}$ is an open set. Indeed, every point $x$ lies on $(-1 / 2+k, 1 / 2+k)$ for some value of $k$ (except for the endpoints of these intervals, which do not satisfy $p$ to begin with), and the interval $(-1 / 2+k, 1 / 2+k)$ contains exactly $k$ points not on $\llbracket p \rrbracket_{\mathfrak{X}}$. This implies that, if $x \in \llbracket p \rrbracket_{\mathfrak{X}} \cap(-1 / 2+k, 1 / 2+k)$, there is a neighborhood $U$ of $x$ contained in $\llbracket p \rrbracket_{\mathfrak{X}}$ (just avoid the $k$ missing points), which in turn implies that $\llbracket p \rrbracket_{\mathfrak{X}}$ is open.

$\varphi_{3}$ : This holds for a similar reason as above. Indeed, suppose $x \in(-1 / 2+$ $k, 1 / 2+k)$ and $x$ satisfies $s \wedge \neg p$. Then, since there are only $k-1$ other points on this interval satisfying $\neg p$, we can find a neighborhood $U$ of $x$ such that $x$ is the only point on this interval satyisfying $\neg p$. Clearly, every point on $U$ which satisfies $\neg s$ then satisfies $p$ (given that $x$ does not satisfy $\neg s)$ and thus $x \in \llbracket \square(\neg s \rightarrow p) \rrbracket_{\mathfrak{X}}$, as desired.

It follows that $\llbracket \square[f] \bigwedge_{i \leq 3} \varphi_{i} \rrbracket_{\mathfrak{X}}=\mathbb{Q}$, so in particular 0 satisfies the antecedent of Baire but not its consequent. We conclude that 0 does not satisfy Baire, as desired.

2. We can also use quasimodels to see that Baire is not valid in general. In fact, we can do this with a rather small (finite!) quasimodel.

Consider the following Baire-quasimodel:

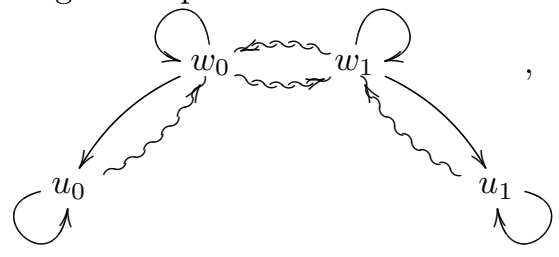


where straight arrows represent $\mapsto$ and squiggly arrows $\succcurlyeq$,

$$
\begin{aligned}
& s, \diamond \neg s, p, \square p, \neg[f] p \quad \in \quad t\left(w_{0}\right) ; \\
& \neg s, \diamond s, p, \square p, \neg[f] p \quad \in t\left(w_{1}\right) ; \\
& s, \square(\neg s \rightarrow p), \neg p \quad \in \quad t\left(u_{0}\right)
\end{aligned}
$$

and

$$
\neg s, \square(s \rightarrow p), \neg p \quad \in \quad t\left(u_{1}\right) .
$$

We let the reader check that

$$
\square[f] \bigwedge_{n \leq 3} \varphi_{n} \in t\left(w_{0}\right)
$$

by inspecting each $\varphi_{n}$, yet note that $\diamond[f] p \notin t\left(w_{0}\right)$.

However, we claim that:

Proposition 8.2. The formula Baire is valid in all complete metric spaces.

Proof. Suppose $\mathfrak{X}$ is a dynamic topological model based on a complete metric space and

$$
\llbracket \square[f] \bigwedge_{n \leq 3} \varphi_{n} \rrbracket_{\mathfrak{X}} \neq \varnothing .
$$

Since this set is open, we can pick out a closed ball $B$ such that

$$
B \subseteq \llbracket[f] \bigwedge_{n \leq 3} \varphi_{n} \rrbracket_{\mathfrak{X}} .
$$

First note that $\varphi_{0}$ implies that both $\llbracket s \rrbracket_{\mathfrak{X}}$ and $\llbracket \neg s \rrbracket_{\mathfrak{X}}$ are dense in $B$, and $\varphi_{1}$ implies that, if $x \in B, x \in \llbracket s \rrbracket_{\mathfrak{X}}$ if and only if $f^{n}(x) \in \llbracket s \rrbracket_{\mathfrak{X}}$ for all $n$. Note also that $\varphi_{2}$ implies that

$$
f_{\mathfrak{X}}^{-n} \llbracket p \rrbracket_{\mathfrak{X}} \cap B
$$

is open for all $n$, because if $x \in f_{\mathfrak{X}}^{-n} \llbracket p \rrbracket_{\mathfrak{X}}$, then

$$
x \in f_{\mathfrak{X}}^{-n} \llbracket \square p \rrbracket_{\mathfrak{X}}
$$

(since $x \in \llbracket[f]\left(p \rightarrow \square p \rrbracket_{\mathfrak{X}}\right.$ ), which is open.

To establish our conclusion, we must also show that $f_{\mathfrak{X}}^{-n} \llbracket p \rrbracket_{\mathfrak{X}}$ is dense in $B$ for all $n$.

Take $x \in B \cap \llbracket s \rrbracket_{\mathfrak{X}}$ and an open ball $B_{\varepsilon}(x)$ around $x$. If $x \in f_{\mathfrak{X}}^{-n} \llbracket p \rrbracket_{\mathfrak{X}}$, there is nothing to prove. Otherwise, $x \in f_{\mathfrak{X}}^{-n} \llbracket \neg p \rrbracket_{\mathfrak{X}}$, so $f_{\mathfrak{X}}^{n}(x) \in \llbracket \neg p \rrbracket_{\mathfrak{X}}$ and hence (by $\varphi_{3}$ )

$$
f_{\mathfrak{X}}^{n}(x) \in \llbracket \square(\neg s \rightarrow p) \rrbracket_{\mathfrak{X}} .
$$


Take a ball $B_{\eta}\left(f_{\mathfrak{X}}^{n}(x)\right)$ such that

$$
B_{\eta}\left(f^{n}(x)\right) \subseteq \llbracket \neg s \rightarrow p \rrbracket_{\mathfrak{X}}
$$

and a neighborhood $B_{\delta}(x)$ of $x$ such that

$$
f_{\mathfrak{X}}^{n}\left(B_{\delta}(x)\right) \subseteq B_{\eta}\left(f_{\mathfrak{X}}^{n}(x)\right)
$$

(such a $\delta$ exists because $f_{\mathfrak{X}}^{n}$ is continuous). Since $\llbracket \neg s \rrbracket$ is dense in $B$, we can pick

$$
y \in \llbracket \neg s \rrbracket_{\mathfrak{X}} \cap B_{\delta}(x) .
$$

But $f_{\mathfrak{X}}^{n}(y) \in B_{\eta}\left(f_{\mathfrak{X}}^{n}(x)\right)$, and $f_{\mathfrak{X}}^{n}(y) \in \llbracket \neg s \rrbracket_{\mathfrak{X}}$, so that $f_{\mathfrak{X}}^{n}(y) \in \llbracket p \rrbracket_{\mathfrak{X}}$, as desired. Since $x$ and $\varepsilon$ were arbitrary, we conclude that $f_{\mathfrak{X}}^{-n} \llbracket p \rrbracket_{\mathfrak{X}}$ is dense for all $n$.

Thus, $f_{\mathfrak{X}}^{-n} \llbracket p \rrbracket_{\mathfrak{X}}$ is open and dense for all $n$. We can now apply the Baire category theorem to show that

$$
\llbracket[f] p \rrbracket_{\mathfrak{X}}=\bigcap_{n \geq 0} f_{\mathfrak{X}}^{-n} \llbracket p \rrbracket_{\mathfrak{X}}
$$

is also dense in $B$ which, being a closed subset of $X$, is a complete metric space on its own right. This means that

$$
B \subseteq \llbracket \triangleright[f] p \rrbracket_{\mathfrak{X}},
$$

and $\mathfrak{X} \models \varphi$.

Corollary 8.1. $\mathcal{D} \mathcal{T} \mathcal{L} \subsetneq \mathcal{D} \mathcal{T} \mathcal{L}_{\text {CompMH }}$.

\section{Universality of the Cantor space}

In this section we will show that any formula that is satisfiable on CompM is satisfiable on a model based on the Cantor space. We will use 'neighborhood trees', a variant of a technique which has been used in $[\underline{4,} \underline{12]}$ for other relative completeness proofs. However, the novelty here is that we do not construct full bisimulations, but rather a rough approximation of a bisimulation called a $\varphi$-simulation which does not preserve the truth of all formulas, but it does preserve subformulas of $\varphi$.

First we state a very useful result which gives a general characterization of spaces homeomorphic to the Cantor space:

Theorem 9.1 (Brouwer). A topological space is a Cantor space if and only if it is non-empty, perfect, compact, totally disconnected, and metrizable.

A topological space is totally disconnected if it has a basis of clopen sets. 
Definition 9.1 (Neighborhood tree). Let $\mathfrak{X}$ be a complete metric space, $N \leq \omega$ and $M<\omega$. A neighborhood tree on $\mathfrak{X}$ of depth $N$ and delay $M$ is a structure $\mathfrak{T}=\left\langle|\mathfrak{T}|, \preccurlyeq \mathfrak{T}, \vartheta_{\mathfrak{T}}, \varepsilon_{\mathfrak{T}}\right\rangle$ such that $\langle|\mathfrak{T}|, \preccurlyeq \mathfrak{T}\rangle$ is a tree,

$$
\vartheta_{\mathfrak{T}}:|\mathfrak{T}| \rightarrow|\mathfrak{X}|
$$

and

$$
\varepsilon_{\mathfrak{T}}:|\mathfrak{T}| \rightarrow[0, \infty]
$$

and such that

1. if $s \preccurlyeq t$ then $\overline{B_{\varepsilon_{\mathfrak{T}}(s)}(\vartheta(s))} \subseteq B_{\varepsilon_{\mathfrak{T}}(t)}\left(\vartheta_{\mathfrak{T}}(t)\right)$;

2. if t has depth $n>M$, then $\varepsilon_{\mathfrak{T}}(t)<1 / 2^{n}$,

3. if $t$ has depth $n<N$, then it has a designated daughter $t^{+}$with $\vartheta_{\mathfrak{T}}\left(t^{+}\right)=$ $\vartheta_{\mathfrak{T}}(t)$

4. if $t$ has depth $n<N$, it has at least two daughters and

5. every node is at depth at most $N$.

As usual, we omit the subindex $\mathfrak{T}$ to avoid cluttering notation whenever this does not lead to confusion. In the end we will be interested in neighborhood trees of depth $\omega$; trees of finite depth will serve as a sort of approximation.

Given a dynamic neighborhood tree $\langle\mathfrak{T}, \vartheta, \varepsilon\rangle$ of depth $\omega$ for $\mathfrak{X} \in$ CompM, we define a space $\widehat{\mathfrak{T}}$ by letting $|\widehat{\mathfrak{T}}|$ be the set of all maximal chains on $\mathfrak{T}$, and define a map

$$
\widehat{\vartheta}:|\widehat{\mathfrak{T}}| \rightarrow|\mathfrak{X}|
$$

given by

$$
\widehat{\vartheta}(\vec{t})=\lim _{n \rightarrow \infty} \vartheta\left(t_{n}\right) .
$$

Note that $\widehat{\vartheta}$ is defined everywhere since $\left\langle\vartheta\left(t_{n}\right)\right\rangle_{n<\omega}$ is Cauchy.

The topology on $\widehat{\mathfrak{T}}$ is generated by basic sets of the form

$$
D_{N}(\vec{t})=\left\{\vec{s} \in|\widehat{\mathfrak{T}}|: s_{N}=t_{N}\right\},
$$

for $\vec{t} \in|\widehat{\mathfrak{T}}|$.

Given $t \in|\mathfrak{T}|$, we will write $t^{\infty}=\left\langle s_{n}\right\rangle_{n<\omega}$ for the branch on $\mathfrak{T}$ which passes through $t$ and such that $s_{n+1}=s_{n}^{+}$for all $n$ greater than the depth of $t$.

We then have that:

Lemma 9.1. If $\mathfrak{T}$ is a neighborhood tree of depth $\omega$, then $\widehat{\mathfrak{T}}$ is homeomorphic to the Cantor space.

Proof. We use Theorem 9.1. The topology on $\widehat{\mathfrak{T}}$ is second-countable since its basic sets $D_{N}(\vec{t})$ can clearly be identified with the node $t_{N}$, because

$$
D_{N}(\vec{t})=\left\{\vec{s}: s_{N}=t_{N}\right\} .
$$


But there are countably many nodes, as desired.

The sets $D_{N}(\vec{t})$ are closed, since their complement is a union of basic sets, namely

$$
\bigcup\left\{D_{N}(\vec{s}): s_{N} \neq t_{N}\right\} .
$$

Therefore, $\widehat{\mathfrak{T}}$ is totally disconnected.

To see that it is perfect, let $\vec{t} \in|\widehat{\mathfrak{T}}|$ and $D_{N}(\vec{t})$ be a basic neighborhood of $\vec{t}$. Then, by condition $4, t_{N}$ has at least two daughers, so at least one is distinct from $t_{N+1}$; call it $s$. Clearly $s^{\infty} \in D_{N}(\vec{t})$ and $s^{\infty} \neq \vec{t}$, as desired.

$\widehat{\mathfrak{T}}$ can be assigned a metric in a fairly standard way, by letting $d(\vec{s}, \vec{t})=1 / 2^{N}$, where $N$ is the least integer such that $t_{N} \neq s_{N}$.

Once we have metrizability we know that compactness is equivalent to sequential compactness (that is, every sequence has a converging subsequence). But this follows from a standard diagonalization argument: Let $\left\langle\vec{t}^{n}\right\rangle_{n<\omega}$ be a sequence of elements of $|\widehat{\mathfrak{T}}|$; we will find a limit point $\vec{s}$ for it. Let $s_{0}$ be the root of $\mathfrak{T}$. Then, since there are only finitely many values that $t_{1}^{n}$ could take, one of them must occur infinitely often, and we take that to be $s_{1}$. From those elements of $\left\langle\vec{t}^{n}\right\rangle_{n<\omega}$ that have $t_{1}^{n}=s_{1}$, infinitely many of them must take on the same value of $t_{2}^{n}$, and we let that be $s_{2}$. Continuing in this fashion we construct $\vec{s}$, which is clearly a limit point of $\left\langle\vec{t}^{n}\right\rangle_{n<\omega}$.

Lemma 9.2. If $\mathfrak{T}$ is a neighborhood tree of depth $\omega$ and $\vec{t} \in|\mathfrak{T}|$, then for all $n<\omega$,

$$
\widehat{\vartheta}(\vec{t}) \in B_{\varepsilon\left(t_{n}\right)}\left(\vartheta\left(t_{n}\right)\right) .
$$

Proof. By definition we have that, for all $i<\omega$,

$$
\overline{B_{\varepsilon\left(t_{i+1}\right)}\left(\vartheta\left(t_{i+1}\right)\right)} \subseteq B_{\varepsilon\left(t_{i}\right)}\left(\vartheta\left(t_{i}\right)\right) .
$$

Now, $\vartheta\left(t_{i}\right) \in B_{\varepsilon\left(t_{n+1}\right)}\left(\vartheta\left(t_{n+1}\right)\right)$ for all $i>n$, which implies that

$$
\widehat{\vartheta}(\vec{t}) \in \overline{B_{\varepsilon\left(t_{n+1}\right)}\left(\vartheta\left(t_{n+1}\right)\right)},
$$

from which it follows that

$$
\widehat{\vartheta}(\vec{t}) \in B_{\varepsilon\left(t_{n}\right)}\left(\vartheta\left(t_{n}\right)\right) .
$$

Definition 9.2. Given a formula $\varphi$ and a neighborhood tree $\mathfrak{T}$ of depth $N \leq \omega$ on a DTM $\mathfrak{X}$, we say that $\mathfrak{T}$ is $\varphi$-preopen if, whenver $\psi \in \operatorname{sub}(\varphi)$ and $t \in|\mathfrak{T}|$ are such that $\llbracket \psi \rrbracket_{\mathfrak{X}} \cap B_{\varepsilon(t)} \vartheta(t) \neq \varnothing$, then $t$ has a daughter $t^{\psi}$ with $\vartheta\left(t^{\psi}\right) \in \llbracket \psi \rrbracket_{\mathfrak{X}}$.

Definition 9.3 ( $\varphi$-open map). Given a formula $\varphi$ and DTM's $\mathfrak{X}$ and $\mathfrak{Y}$, we say a function

$$
\vartheta:|\mathfrak{X}| \rightarrow|\mathfrak{Y}|
$$

is $\varphi$-open if, given $\psi \in \operatorname{sub}(\varphi)$ and $x \in \vartheta^{-1} \llbracket \triangleright \psi \rrbracket_{\mathfrak{Y}}$, every neighborhood $U$ of $x$ contains a point $y$ such that $y \in \vartheta^{-1} \llbracket \psi \rrbracket_{\mathfrak{Y}}$. 
The map $\vartheta$ is a $\varphi$-simulation if, in addition, it is continuous and

$$
\vartheta \circ f_{\mathfrak{X}}=f_{\mathfrak{Y}} \circ \vartheta .
$$

Lemma 9.3. If $\vartheta$ is a $\varphi$-simulation between $\mathrm{DTM}$ 's $\mathfrak{X}$ and $\mathfrak{Y}$, for all $\psi \in \operatorname{sub}(\varphi)$, $\llbracket \psi \rrbracket_{\mathfrak{X}}=\vartheta^{-1} \llbracket \psi \rrbracket_{\mathfrak{Y}}$.

Proof. Routine induction on $\psi$.

Definition 9.4 (Dynamic neighborhood tree). A dynamic neighborhood tree of depth $N$ and duration $K \leq \omega$ on a DTM $\mathfrak{X}$ is a sequence of neighborhood trees $\left\langle\mathfrak{T}_{k}\right\rangle_{k<K}$ of depth $N$ equipped with a partial function $g_{\mathfrak{T}}$ defined on all $\left|\mathfrak{T}_{k}\right|$ with $k+1<K$ such that

1. $g_{\mathfrak{T}}\left|\mathfrak{T}_{k}\right| \subseteq\left|\mathfrak{T}_{k+1}\right|$ whenever $k+1<K$;

2. if $g_{\mathfrak{T}}(s), g_{\mathfrak{T}}(t)$ are defined and $s$ is a daughter of $t$ then $g_{\mathfrak{T}}(s)$ is a daughter of $g_{\mathfrak{T}}(t)$;

3. $\mathfrak{T}_{k}$ has delay at most $k$ and

4. if $g_{\mathfrak{T}}(t)$ is defined then

$$
f_{\mathfrak{X}} B_{\varepsilon(t)}(\vartheta(t)) \subseteq B_{\varepsilon(g \mathfrak{I}(t))}\left(\vartheta\left(g_{\mathfrak{T}}(t)\right)\right) .
$$

Lemma 9.4. Let $\vartheta:|\mathfrak{T}| \rightarrow|\mathfrak{X}|$ be a neighbornood tree of finite depth $N$ and duration $N, t \in\left|\mathfrak{T}_{k}\right|$ and $x \in B_{\varepsilon(t)}(\vartheta(t))$.

Then, there exists an extension $\mathfrak{T}^{\prime}$ of $\mathfrak{T}$ such that $t$ has a daughter $s$ on $\mathfrak{T}^{\prime}$ with $\vartheta(s)=x$.

Proof. First, add a daughter $s$ to $t$ and daughters $g^{i}(s)$ to each respective $g^{i}(t)$. Let $M=N-k$ and set

$$
\vartheta\left(g^{i}(s)\right)=f_{\mathfrak{X}}^{i}(x) .
$$

Now, let $\varepsilon\left(g^{M}(s)\right)<1 / 2^{N}$ be small enough so that

$$
\overline{B_{\delta_{N}}\left(f_{\mathcal{X}}^{M}(x)\right)} \subseteq B_{\varepsilon\left(g^{M}(t)\right)}\left(\vartheta\left(g^{M}(t)\right)\right) .
$$

Assume, inductively, that we have defined

$$
\varepsilon\left(g^{M-i}(s)\right), \ldots, \varepsilon\left(g^{M-i}(s)\right) .
$$

Then pick $\varepsilon=\varepsilon\left(g^{M-(i+1)}(s)\right)<1 / 2^{N}$ small enough so that

- $\overline{B_{\varepsilon}\left(\vartheta\left(g^{M-(i+1)}(s)\right)\right)} \subseteq B_{\varepsilon\left(g^{M-(i+1)}(t)\right)}\left(\vartheta\left(g^{M-(i+1)}(t)\right)\right)$

- $f_{\mathfrak{X}} B_{\varepsilon}\left(\vartheta\left(g^{M-(i+1)}(s)\right)\right) \subseteq B_{\varepsilon\left(g^{M-i}(s)\right)}\left(\vartheta\left(g^{M-i}(s)\right)\right)$.

Continuing in this way we can define

$$
\varepsilon(s), \varepsilon(g(s)), \ldots, \varepsilon\left(g^{N-k}(s)\right) .
$$

It is clear that $\vartheta, \varepsilon$ satisfy the required conditions. 
Lemma 9.5. Let $\mathfrak{T}$ be a $\varphi$-preopen dynamic neighborhood tree of finite depth $N$ and duration $N$ on a DTM $\mathfrak{X}$. Then, $\mathfrak{T}$ can be extended to a $\varphi$-preopen dynamic neighborhood tree of depth $N+1$ and duration $N+1$ without altering any nodes of depth less than $N$ at time less than $N$.

Proof. We begin by extending each $\mathfrak{T}_{k}$ to a tree with depth $N+1$. Our strategy will be to add one node at a time until we obtain the desired extension, by removing all 'problems'.

A problem is an occurrence of a node $t \in\left|\mathfrak{T}_{m}\right|$ of depth $N$ such that either

1. $t$ does not have a designated successor $t^{+}$with $\vartheta\left(t^{+}\right)=\vartheta(t)$;

2. $t$ only has one daughter or

3. there is $\psi \in \operatorname{sub}(\varphi)$ such that $\llbracket \psi \rrbracket_{\mathfrak{X}} \cap B_{\varepsilon(x)}(\vartheta(t)) \neq \varnothing$ but there is no daughter $s$ of $t$ with $\vartheta(s) \in \llbracket \psi \rrbracket$.

Since the trees are all finitely branching and there are finitely many of them, we have finitely many nodes on them, and hence finitely many problems. It is clear that if we eliminate all problems we have the desired extension; since there are finitely many problems, we will show only how to remove one of them at at time.

1. If $t$ does not have a designated successor $t^{+}$with $\vartheta\left(t^{+}\right)=\vartheta(t)$, then we can use Lemma 9.4 with $x=\vartheta(t)$ to define $t^{+}$.

2. If $t$ only has one daughter, duplicate

$$
t^{+}, g\left(t^{+}\right), g^{2}\left(t^{+}\right), \ldots, g^{N-m}\left(t^{+}\right)
$$

without changing $\vartheta$ or $\varepsilon$.

3. If there is $\psi \in \operatorname{sub}(\varphi)$ such that $\llbracket \psi \rrbracket_{\mathfrak{X}} \cap B_{\varepsilon(x)}(\vartheta(t)) \neq \varnothing$ but there is no daughter $s$ of $t$ with $\vartheta(s) \in \llbracket \psi \rrbracket$, pick $x \in \llbracket \psi \rrbracket_{\mathfrak{X}} \cap B_{\varepsilon(x)}(\vartheta(t))$ and use Lemma 9.4 to add a node $t^{\psi}$ with $\vartheta\left(t^{\psi}\right)=x$.

In this way we can remove problems one at a time and obtain an extension $\mathfrak{T}^{\prime \prime}$ of depth $N+1$.

Now, to obtain $\mathfrak{T}^{\prime}$, we will extend $\mathfrak{T}^{\prime \prime}$ so that it has duration $N+1$. But indeed this is not hard to do; just define a tree $\mathfrak{T}_{N}^{\prime}$ by taking a copy of $\mathfrak{T}_{N-1}^{\prime \prime}$ of the form $\left\{g(t): t \in\left|\mathfrak{T}_{N-1}^{\prime \prime}\right|\right\}$ and setting $\vartheta(g(t))=f_{\mathfrak{X}}(\vartheta(t)), \varepsilon(g(t))=\infty$. Note that the new nodes are at time $N$ but have depth at most $N$, so we do not have any restriction on $\varepsilon$.

The resulting dynamic neighborhood $\mathfrak{T}^{\prime}$ then has depth and duration $N+1$, as desired.

Lemma 9.6. If $\mathfrak{X} \in \mathrm{M}, \varphi$ is any formula and $x_{*} \in|\mathfrak{X}|$, there exists a $\varphi$-preopen dynamic tree $\mathfrak{T}$ on $\mathfrak{X}$ with $x_{*} \in \operatorname{im}(\vartheta)$. 
Proof. First define $\mathfrak{T}_{0}$ as a dynamic neighborhood tree of depth and duration 1 (i.e., an ordinary neighborhood tree) with a single node $t_{*}$ and $\vartheta\left(t_{*}\right)=x_{*}$, $\varepsilon\left(t_{*}\right)=\infty$.

We can then use Lemma 9.5 countably many times to generate an increasing chain $\left\langle\mathfrak{T}_{n}\right\rangle_{n<\omega}$, where $\mathfrak{T}_{n}$ has depth and duration $n$. Then, $\mathfrak{T}_{\omega}$ defined by taking

$$
\left|\mathfrak{T}_{\omega}\right|=\bigcup_{n<\omega}\left|\mathfrak{T}_{n}\right|
$$

is the desired dynamic tree of depth $\omega$.

The dynamic neighborhood tree $\mathfrak{T}$ we have constructed above will give us our desired model based on the Cantor space by taking (an extension of) $\widehat{\mathfrak{T}}$. It remains to check that $\widehat{\mathfrak{T}}$ truly is a DTM satisfying $\varphi$, as will be seen in the following lemmas.

First let us consider the dynamics on $\widehat{\mathfrak{T}}$. We define $\widehat{g}_{\mathfrak{T}}$ by

$$
\widehat{g}_{\mathfrak{T}}\left\langle t_{n}\right\rangle_{n<\omega}=\left\langle g_{\mathfrak{T}}\left(t_{n}\right)\right\rangle_{n<\omega} .
$$

Lemma 9.7. If $\mathfrak{T}$ is a neighborhood tree of depth $\omega$, then $\widehat{g}_{\mathfrak{T}}$ is a continuous function mapping $\widehat{\mathfrak{T}}$ into itself. In particular,

$$
\widehat{g}_{\mathfrak{T}}\left|\widehat{\mathfrak{T}}_{n}\right| \subseteq\left|\widehat{\mathfrak{T}}_{n+1}\right| .
$$

Proof. We know that $g_{\mathfrak{T}}$ preserves daughters, so it maps maximal chains to maximal chains, i.e., $\operatorname{im}|\widehat{\mathfrak{T}}| \subseteq|\widehat{\mathfrak{T}}|$. Because $g_{\mathfrak{T}}\left|\mathfrak{T}_{n}\right| \subseteq\left|\widehat{\mathfrak{T}_{n+1}}\right|$ for all $n$, we also have that $\widehat{g}_{\mathfrak{T}}\left|\widehat{\mathfrak{T}}_{n}\right| \subseteq\left|\widehat{\mathfrak{T}}_{n+1}\right|$.

It remains to check that $\widehat{g}_{\mathfrak{T}}$ is continuous, but this is not hard to do; we need only check that the preimage of any basic set around $\widehat{g}_{\mathfrak{T}}(\vec{t})$ contains a basic set around $\vec{t}$. So, let $D_{N}\left(\widehat{g}_{\mathfrak{T}}(\vec{t})\right)$ be such a basic set; then, clearly

$$
D_{N}(\vec{t}) \subseteq g_{\mathfrak{T}}^{-1} D_{N}\left(\widehat{g}_{\mathfrak{T}}(\vec{t})\right)
$$

We conclude that

$$
\widehat{g}_{\mathfrak{T}}:|\widehat{\mathfrak{T}}| \rightarrow|\widehat{\mathfrak{T}}|
$$

is continuous, as desired.

Lemma 9.8. If $\mathfrak{T}$ is a dynamic tree, $\vec{t} \in\left|\widehat{\mathfrak{T}}_{m}\right|$ and $N>m$, then

$$
d\left(\vartheta\left(t_{N}\right), \widehat{\vartheta}(\vec{t})\right)<1 / 2^{N-1}
$$

Proof. Note that for $n>N$ we have that $d\left(\vartheta\left(t_{n}\right), \vartheta\left(t_{n}\right)\right)<1 / 2^{-n}$, and since we 
have that $\widehat{\vartheta}(\vec{t})=\lim _{n \rightarrow \infty} \vartheta\left(t_{n}\right)$, we can compute

$$
\begin{aligned}
d\left(\widehat{\vartheta}(\vec{t}), \vartheta\left(t_{N}\right)\right) & \left.\leq d\left(\widehat{\vartheta}(\vec{t}), \vartheta\left(t_{N}\right)\right) \vartheta(\vec{t})\right) \\
& \leq \sum_{n=N}^{\infty} d\left(\widehat{\vartheta}\left(t_{n}\right), \widehat{\vartheta}\left(t_{n+1}\right)\right) \\
& \leq \sum_{n=N}^{\infty} 1 / 2^{n} \\
& =1 / 2^{N-1} .
\end{aligned}
$$

Lemma 9.9. If $\mathfrak{X} \in \mathrm{CompM}$ and

$$
\vartheta:|\mathfrak{T}| \rightarrow|\mathfrak{X}|
$$

is a neighborhood tree, then $\widehat{\vartheta}$ is a $\varphi$-simulation.

Proof. First let us show that $\widehat{\vartheta}$ is continuous.

In fact, the continuity of $\widehat{\vartheta}$ is uniform in the following sense: if $\vec{t}, \vec{s} \in\left|\widehat{\mathfrak{T}}_{m}\right|$, $N>m$ and $s_{n}=t_{n}$ for all $n<N$, then $d(\widehat{\vartheta}(\vec{s}), \widehat{\vartheta}(\vec{t})) \leq 1 / 2^{N-2}$.

To see this, note that

$$
d(\widehat{\vartheta}(\vec{s}), \widehat{\vartheta}(\vec{t})) \leq d\left(\widehat{\vartheta}(\vec{s}), \vartheta\left(t_{K}\right)\right)+d\left(\vartheta\left(t_{K}\right), \widehat{\vartheta}(\vec{t})\right) .
$$

But by Lemma 9.8, this is at most

$$
1 / 2^{K-1}+1 / 2^{K-1}=1 / 2^{K-2} .
$$

Now it remains to show that $\widehat{\vartheta}$ is $\varphi$-open. Let $\vec{t} \in|\mathfrak{T}|$ and suppose that $\widehat{\vartheta}(\vec{t}) \in \llbracket \diamond \psi \rrbracket_{\mathfrak{X}}$ for some $\psi \in \operatorname{sub}(\varphi)$.

Then, for any neighborhood $U$ of $\vec{t}$ there is $N>0$ such that $D_{N}(\vec{t}) \subseteq U$.

Now, $B_{\varepsilon\left(t_{N}\right)}\left(\vartheta\left(t_{N}\right)\right)$ is a neighborhood of $\widehat{\vartheta}(\vec{t})$ by Lemma 9.2, and since $\widehat{\vartheta}(\vec{t})$ satisfies $\diamond \psi$ it follows that

$$
B_{\varepsilon\left(t_{N}\right)}\left(\vartheta\left(t_{N}\right)\right) \cap \llbracket \psi \rrbracket_{\mathfrak{X}} \neq \varnothing .
$$

Hence $t_{N}$ has a daughter $s$ with $\vartheta(s) \in \llbracket \psi \rrbracket_{\mathfrak{X}}$, and thus

$$
s^{\infty} \in D_{N}(\vec{t}) \cap \widehat{\vartheta}^{-1} \llbracket \psi \rrbracket_{\mathfrak{X}},
$$

as desired

Finally, we need to check that

$$
f_{\mathfrak{X}} \circ \widehat{\vartheta}=\vartheta \circ \widehat{g}
$$

but this is an easy consequence of the fact that $f_{\mathfrak{X}} \circ \vartheta=\vartheta \circ g$. 
Lemma 9.10. Let $f$ be a continuous function on $\coprod_{n<\omega} \mathbb{K}$ such that $f\left(\mathbb{K}_{n}\right) \subseteq$ $\mathbb{K}_{n+1}$ for all $n$, where $\mathbb{K}_{n}$ is the $n^{\text {th }}$ copy of $\mathbb{K}$.

Then, there is an open, continuous injection

$$
\iota: \coprod_{n<\omega} \mathbb{K} \rightarrow \mathbb{K}
$$

and a continuous map $f^{+}$extending $f$, that is, such that

$$
f=f^{+} \circ \iota \text {. }
$$

Proof. Consider the space

$$
\mathfrak{Z}=\{\infty\} \cup \coprod_{n<\omega} \mathbb{K}
$$

where $\infty$ is a fresh point and neighborhoods of $\infty$ are of the form $\{\infty\} \cup$ $\bigcup_{n>N} \mathbb{K}_{n}$.

It is not hard to see that $\mathfrak{Z}$ is a Cantor space containing $\coprod_{n<\omega} \mathbb{K}$, and we can extend $f$ by setting $f(\infty)=\infty$.

\section{Theorem 9.2.}

$$
\mathcal{D} \mathcal{T} \mathcal{L}_{\mathbb{K}}=\mathcal{D} \mathcal{T} \mathcal{L}_{\text {CompM }}
$$

Proof. Clearly $\mathcal{D} \mathcal{T} \mathcal{L}_{\mathbb{K}} \supseteq \mathcal{D} \mathcal{T} \mathcal{L}_{\text {CompM }}$, so we will focus on the other direction.

Let $\varphi$ be a formula and suppose that $\varphi$ is satisfiable on CompM. Let us show that it can also be satisfied on $\mathbb{K}$.

Let $\mathfrak{X} \in$ CompM satisfy $\varphi$ and $x_{*} \in \llbracket \varphi \rrbracket_{\mathfrak{X}}$.

By Lemma 9.6 there is a dynamic tree $\mathfrak{T}$ with a $\varphi$-open Cauchy map $\vartheta$ : $|\mathfrak{T}| \rightarrow|\mathfrak{X}|$ such that $x_{*} \in \operatorname{im}(\widehat{\vartheta})$.

By Lemma 9.9 , this then gives us a $\varphi$-simulation $\widehat{\vartheta}:|\widehat{\mathfrak{T}}| \rightarrow|\mathfrak{X}|$.

Now, setting $\llbracket \cdot \rrbracket_{\widehat{\mathfrak{T}}}=\widehat{\vartheta}^{-1} \llbracket \cdot \rrbracket_{\mathfrak{X}}$ and using Lemma 9.3, we see that, for $\vec{t} \in$ $\widehat{\vartheta}^{-1}\left(x_{*}\right), \vec{t} \in \llbracket \varphi \rrbracket_{\widehat{\mathfrak{T}}}$.

Finally, we use Lemma 9.10 to embed $\widehat{\mathfrak{T}}$ into a DTM $\mathfrak{Y}$ based on $\mathbb{K}$ via an inclusion $\iota$. Setting $\llbracket \cdot \rrbracket_{\mathfrak{Y}}=\iota \llbracket \cdot \rrbracket_{\mathfrak{T}}$, it follows that $\mathfrak{Y}$ satisfies $\varphi$, as desired.

\section{References}

[1] E. Akin. The General Topology of Dynamical Systems. Graduate Studies in Mathematics. American Mathematical Society, 1993.

[2] S.N. Artemov, J.M. Davoren, and A. Nerode. Modal logics and topological semantics for hybrid systems. Technical Report MSI 97-05, 1997.

[3] D. Fernández-Duque. Dynamic topological logic interpreted over minimal systems. Journal of Philosophical Logic. Forthcoming. 
[4] D. Fernández-Duque. Dynamic topological completeness for $\mathbb{R}^{2}$. Logic Journal of IGPL, 2007. doi: 10.1093/jigpal/jzl036.

[5] D. Fernández-Duque. Non-deterministic semantics for dynamic topological logic. Annals of Pure and Applied Logic, 157(2-3):110-121, 2009. Kurt Gödel Centenary Research Prize Fellowships.

[6] P. Kremer. The modal logic of continuous functions on the rational numbers. Archive for Mathematical Logic, 49(4):519-527, 2010.

[7] P. Kremer and G. Mints. Dynamic topological logic. Annals of Pure and Applied Logic, 131:133-158, 2005.

[8] O. Lichtenstein and A. Pnueli. Propositional temporal logics: Decidability and completeness. Logic Jounal of the IGPL, 8(1).

[9] G. Mints and T. Zhang. Propositional logic of continuous transformations in cantor space. Archive for Mathematical Logic, 44:783-799, 2005.

[10] M. Nogin and A. Nogin. On dynamic topological logic of the real line. Journal of Logic and Computation, 18(6):1029-1045, 2008. doi:10.1093/logcom/exn034.

[11] S. Slavnov. Two counterexamples in the logic of dynamic topological systems. Technical Report TR-2003015, 2003.

[12] S. Slavnov. On completeness of dynamic topological logic. Moscow Mathematical Journal, 5(2), 2005.

[13] A. Tarski. Der aussagenkalkül und die topologie. Fundamenta Mathematica, 31:103-134, 1938. 